\title{
Reparative properties of the traditional Chinese medicine Cordyceps sinensis (Chinese caterpillar mushroom) using HT29 cell culture and rat gastric damage models of injury
}

\author{
Tania Marchbank, Ehighale Ojobo, Christopher J. Playford and Raymond J. Playford* \\ Centre for Digestive Diseases, Blizard Institute of Cell and Molecular Science, Barts and The London School of Medicine and \\ Dentistry, Queen Mary's University of London, Turner Street, Whitechapel, London E1 2AD, UK
}

(Received 5 July 2010 - Revised 19 October 2010 - Accepted 9 November 2010 - First published online 28 January 2011)

\section{Abstract}

Cordyceps sinensis (CS) is a traditional Chinese medicine and health food used to support many organ systems. It is commercially produced by cultivation in a liquid medium or on a solid (grain/potato) phase. We tested the effects of hot water extracts of liquid-phase and solidphase commercially grown CS on its ability to influence proliferation (using Alamar blue, an oxidation/reduction indicator), migration (serial-wounded monolayer photomicroscopy), invasion through collagen gel (fluorometric assay) and indomethacin-induced apoptosis (active caspase-3 colorimetric assay) of human colon cancer HT29 cells. An in vivo study used a rat gastric damage model (indomethacin $20 \mathrm{mg} / \mathrm{kg}$ and $4 \mathrm{~h}$ restraint with oral administration). The CS extract stimulated cell proliferation threefold when added at $10 \mu \mathrm{g} / \mathrm{ml}$ $(P<0 \cdot 01)$. Cell migration increased by $69 \%$ and invasion by $17 \%$ when CS was added at $5 \mathrm{mg} / \mathrm{ml}(P<0 \cdot 01)$. The results also showed that $93 \%$ of the pro-proliferative activity was soluble in ethanol, whereas pro-migratory activity was divided (61:49) into both ethanolsoluble and ethanol-insoluble sub-fractions. Indomethacin-induced apoptosis was not affected by the presence of CS. CS reduced the amount of gastric injury by $63 \%$ when administered orally at $20 \mathrm{mg} / \mathrm{ml}(P<0 \cdot 01)$, the results being similar to using the potent cytoprotective agent epidermal growth factor at $25 \mu \mathrm{g} / \mathrm{ml}$ ( $83 \%$ reduction). We conclude that both methods of cultivated CS possess biological activity when analysed using a variety of gut models of injury and repair. Functional foods, such as CS, could provide a novel approach for the prevention and treatment of injury to the bowel.

\section{Key words: Nutriceuticals: Repair: Gut growth: Injury}

Natural medicinal products have been used for millennia for the treatment of multiple ailments. Although many have been superseded by conventional pharmaceutical approaches, there is currently a resurgence of interest in the use of natural bioactive products by the general public for the prevention and treatment of multiple conditions, including gastrointestinal disorders ${ }^{(1)}$. Unfortunately, current evidence of the scientific validity of many of these traditional and commercial compounds is severely limited.

Cordyceps is a genus of ascomycete fungi (sac fungi) that includes over 400 described species. All Cordyceps species are endoparasitoids, mainly on insects and other arthropods. Cordyceps sinensis (CS) is known commonly as Chinese caterpillar mushroom and grows on the larvae of the Lepidoptera, Thitarodes namnai, the ghost moth. It is considered to possess therapeutic properties in traditional Chinese and Tibetan Medicines. Wild Cordyceps is a black, blade-shaped fungus found mainly above $4000 \mathrm{~m}$ in a mountainous region of China known as the Qinghai-Tibetan Highlands. CS takes about 6 years to complete its life cycle, and wild-sourced CS is, therefore, both rare and expensive. Because of prohibitive costs, industrially grown CS is the form normally used. Two different methods are commercially used for the cultivation of CS. Liquid culture (also known as fermentation) is a method where the fungus is grown in a sterilised liquid medium. The mycelium is subsequently harvested by straining and drying and can be further processed. Using this method, extracellular compounds are usually discarded with the spent broth. An alternative method employs cultivating the mycelium in plastic bags or glass jars containing some type of cereal grain, usually rice, wheat or rye $\mathrm{e}^{(2,3)}$.

There have been many health claims that this product possesses beneficial effects against ageing, tumour growth, improved immune function, gut health, cholesterol lowering,

Abbreviations: CS, Cordyceps sinensis; DMEM, Dulbecco's modified Eagle's medium; FCS, fetal calf serum.

*Corresponding author: Professor R. J. Playford, fax + 44207377 7607, email r.playford@qmul.ac.uk 
enhanced blood flow, exercise performance and fatigue recovery $^{(4-8)}$. However, as with most products of a complementary background, the data underlying such claims are weak. Nevertheless, pharmacologically important materials, such as cyclosporin, have been isolated previously from the related anamorph of Cordyceps subsessilis.

In order to further examine its potential biological actions in a scientific setting, we have, therefore, performed a series of studies to analyse liquid-phase- and solid-phase-grown CS with regard to their effects on various mechanisms of gut integrity and repair using well-validated in vitro (proliferation, restitution, invasion and apoptosis assays) and in vivo models (indomethacin- and restraint-induced gastric injury).

\section{Materials and methods}

All chemicals were purchased from Sigma (Poole, Dorset, UK), unless otherwise stated.

\section{Cell line}

HT29 ${ }^{(9)}$ cells were derived from a 44-year-old Caucasian female suffering from colonic adenocarcinoma (LGC, Teddington, Middlesex, UK).

\section{Ethics}

All animal studies were approved by appropriate regulatory authorities. CS was sourced from China. Whole-dried fruiting bodies grown on a rice and potato medium were obtained from producers (Xin Hui Jin Gu Zhou Development Area, Guangdong Province, Jiangmen City, China), and a powdered dried extract from the mycelium grown in liquid culture, CS- 4 (batch 0901053), was sourced from importers (Cambridge Commodities Limited, Ely, Cambridge, UK).

\section{Preparation of the extracts of Cordyceps sinensis}

Various extracts of CS were tested, which are as follows.

(1) Whole rice- and potato-grown hot water extract. Whole fruiting bodies were boiled in distilled water for $30 \mathrm{~min}$. The liquid was decanted from the fruiting bodies and cleared by centrifugation at $3000 \mathrm{~g}$ for $10 \mathrm{~min}$ at $4^{\circ} \mathrm{C}$. This sample reproduced how the product is commonly prepared by individuals.

(2) Ground-rice- and potato-grown hot water extract. Dried fruiting bodies were ground to a fine powder using a pestle and mortar. The ground powder was heated in distilled water at $90^{\circ} \mathrm{C}$ for $2 \mathrm{~h}$, and left to cool to room temperature. The cooled mixture was cleared by centrifugation at $3000 \mathrm{~g}$ for $10 \mathrm{~min}$ at $4^{\circ} \mathrm{C}$. This sample was produced to maximise the recovery of water-soluble material.

(3) Powdered liquid medium-grown bot water extract. The CS extract powder was extracted as described in (2) above. This allowed comparison of rice- and potatogrown and liquid medium-grown forms.
(4) In order to start to examine possible active components, we performed a further step on some of the material produced under (2) above. For these samples, the whole-ground rice- and potato-grown hot water extract was stirred with ethanol $(1: 5, \mathrm{v} / \mathrm{v})$ at $4^{\circ} \mathrm{C}$ for $4 \mathrm{~h}$. The resulting precipitate was resuspended in distilled water (extract 4A, ethanol insoluble). The liquid phase was decanted and then dried using a water-bath and condenser under vacuum; the resultant material was resuspended in distilled water (extract $4 \mathrm{~B}$, ethanol soluble).

The protein concentration of the various CS extracts was determined using a standard Pierce bicinchoninic acid protocol $^{(10)}$.

\section{Assay protocols}

\section{Effect of Cordyceps sinensis on cell migration and invasion in vitro}

Background. An early repair response following injury to tissue is migration of surviving cells over any denuded area to re-establish epithelial integrity. To reproduce this process, cell culture models are commonly used as surrogate markers of this pro-migratory response. Two related models were used, one analysing the effect of CS on migration of cells across wounded monolayers and the other on its effect on the invasion of cells through extracellular matrices. The concentration range used for the in vitro studies was determined based on a pilot study showing that concentrations $>20 \mathrm{mg} / \mathrm{ml}$ induced toxicity, as demonstrated by increased permeability of the cells to the vital dye Trypan blue (data not shown).

Cell migration protocol. Cell migration assays were performed using our previously published methods and equipment ${ }^{(11)}$, using the human colonic carcinoma cell line HT29. Briefly, cells were grown to confluence in six-well plates in a medium consisting of Dulbecco's modified Eagle's medium (DMEM) containing 10\% fetal calf serum (FCS) at $37^{\circ} \mathrm{C}$ in $5 \% \mathrm{CO}_{2}$ and were then serum-starved for $24 \mathrm{~h}$. The monolayers were then wounded by scraping a disposable pipette tip across the dishes, washed with a fresh serum-free medium and cultured in a serum-free medium in the presence of various extracts of CS at various concentrations $(0 \cdot 1-10 \mathrm{mg}$ protein $/ \mathrm{ml})$ in a serum-free medium. DMEM $+10 \%$ FCS was used as a positive control, and DMEM alone was used as a negative control. Additional monolayers treated with CS also had the proliferation inhibitor mitomycin $\mathrm{C}$ added at $5 \mu \mathrm{g} / \mathrm{ml}$ to examine whether wound closure was dependent on cell proliferation.

(a) The rate of movement of the anterior edges of the wounded monolayers was determined by taking serial photomicrographs at various times after wounding. Identical regions were examined at each time point by pre-marking the base of the plates to facilitate alignment. Then, twenty measurements per field were performed by placing a transparent grid over the photograph and measuring the distance moved from 
the original wound line. All results are expressed as means with their standard errors of three separate experiments.

(b) Cell invasion protocol. Cell invasion assays were performed using a Cultrex 96 well Collagen I kit (AMS Biotechnology (Europe) Limited, Abingdon, Oxford, UK) as per the manufacturer's instructions. Briefly, HT29 cells in $0.5 \%$ FCS containing DMEM at $1 \times 10^{6}$ cells/ml were seeded in the top chamber on a layer of collagen I. Cells were then incubated in DMEM containing $0.5 \%$ FCS alone (negative control), or also containing CS extract 1 or $2(2.5$ or $5 \mathrm{mg}$ protein $/ \mathrm{ml})$ or containing $10 \%$ FCS (positive control). DMEM was added to the bottom chamber of all wells, and plates were then incubated at $37^{\circ} \mathrm{C}$ for $48 \mathrm{~h}$. The medium was subsequently removed from both the top and bottom chambers, and each well was washed with wash buffer. Calcein AM containing cell dissociation solution was added to each bottom chamber of the wells and kept in the incubator at $37^{\circ} \mathrm{C}$ for $60 \mathrm{~min}$. Plates were read at $485 \mathrm{~nm}$ excitation, and $520 \mathrm{~nm}$ emission using a fluorimeter (Fluostar Optima Plate Reader; BMG Labtech, Aylesbury, Bucks, UK).

Effect of the Cordyceps sinensis extracts on cell proliferation. Cell proliferation assays utilised Alamar blue (Invitrogen, Paisley, UK) $^{(12,13)}$; this assay incorporates a specially selected oxidation-reduction indicator that undergoes colorimetric change in response to cellular metabolic reduction. The assay was performed as per the manufacturer's instructions measuring changes in absorbance at $570 \mathrm{~nm}$.

Briefly, HT29 cells were seeded at 2000 cells/well, grown in DMEM and $10 \%$ FCS in ninety-six-well plates overnight. The following day, cells were washed with DMEM alone and incubated with the various extracts of CS at varying concentrations (0.0001-10 mg protein $/ \mathrm{ml})$ in a serum-free medium. DMEM $+10 \%$ FCS was used as a positive control, and DMEM alone was used as a negative control.

Effect of the Cordyceps sinensis extracts on apoptosis (active caspase-3). Many toxic agents mediate damage through inducing apoptosis. We, therefore, tested the effect of the various CS extracts on the amount of apoptosis seen in a well-validated model using our previously published methods ${ }^{(14)}$.

Briefly, HT29 cells were seeded at $5 \times 10^{5}$ cells/flask in $\mathrm{T} 75 \mathrm{~cm}$ flasks in DMEM containing $10 \% \mathrm{FCS}$, and were grown for $24 \mathrm{~h}$. Cells were then treated for $4 \mathrm{~h}$ with medium containing FCS alone, or also containing indomethacin ( $800 \mu \mathrm{M}$, to stimulate apoptosis), CS extract 1 or $2(5 \mathrm{mg} / \mathrm{ml})$ or indomethacin and the CS extract. Cells were washed in ice-cold PBS, and lysed in lysis buffer (50 mM-HEPES, 5 mm-dithiothreitol, $0 \cdot 1 \mathrm{mm-EDTA}$ and $0.1 \%$ 3-[(3-cholamidopropyl)-dimethylammonio]-1-propane sulfonate; $\mathrm{pH} 7 \cdot 4$ ) for $5 \mathrm{~min}$ on ice. Lysates were cleared by centrifugation at $10000 \mathrm{~g}$ for $10 \mathrm{~min}$ at $4^{\circ} \mathrm{C}$. Protein concentrations were determined using a standard bicinchoninic acid method (Pierce, Rockford, IL, USA). Caspase-3 activity was measured using the caspase-3 cellular activity assay kit (235419;
Merck Chemicals Limited, Nottingham, Nottinghamshire, UK) following the manufacturer's instructions. Recombinant caspase-3 (30 U) was used as a positive control. In total, $100 \mu \mathrm{g}$ of protein was used in triplicate wells for each treatment; additional wells containing cell lysates had a specific caspase-3 inhibitor (N-Ac-Asp-Glu-Val-Asp-CHO; Ac-DEVD$\mathrm{CHO}$ ) added to show that any activity detected was caspase-3 specific. Absorbance at $405 \mathrm{~nm}$ was determined at $10 \mathrm{~min}$ intervals over a $2 \mathrm{~h}$ period.

Effect of the Cordyceps sinensis extracts on gastric damage in vivo

Background. Although cell culture studies have provided valuable information regarding potential bioactivity, additional insight may be gained by extending studies to the in vivo situation. The ability of CS to reduce gastric injury in rats was, therefore, assessed using a well-validated model ${ }^{(15)}$.

Protocol. Briefly, following an overnight fast, SpragueDawley rats ( $n$ 8, 225-275g) were randomised to receive, by gastric administration, one of the following factors: $2 \mathrm{ml}$ of saline (negative control); CS extract 2 or 3 (rice- and potato- or liquid-phase-grown ground hot water extracts at 10 or $20 \mathrm{mg}$ protein $/ \mathrm{ml}$ ); epidermal growth factor (epidermal growth factor, $25 \mu \mathrm{g} / \mathrm{ml}$, positive control). All solutions that were administered orally also contained $2 \%$ hydroxypropyl methylcellulose to delay gastric emptying. At $30 \mathrm{~min}$ after oral administration, all rats received indomethacin (20 mg/kg, subcutaneously) and were placed in Bollmantype restraint cages. After $3 \mathrm{~h}$, animals were killed, their stomachs were removed and the intragastric $\mathrm{pH}$ was determined using a micro $\mathrm{pH}$ electrode. This $\mathrm{pH}$ assessment was performed to determine whether $\mathrm{pH}$ remained below 4 , as $\mathrm{pH}$ rises above this level influence the degree of injury that occurs using this model ${ }^{(16)}$.

The stomachs were then inflated with $4 \mathrm{ml}$ of $10 \%$ neutralbuffered formalin. The following day, the stomachs were opened and placed in fresh formalin before assessment. The stomachs were randomly coded, and all analyses of gastric damage were assessed blind. Macroscopic injury was assessed using a dissecting microscope $(10 \times)$ with the aid of a reference square grid by a trained histopathologist who was blinded to the identity of the tissues. The stomachs were then embedded in wax, and the depth of damage was assessed microscopically, as previously described ${ }^{(15)}$. Using this system, each stomach was given a score from 0 to 4 , where $0=$ no damage, $1=$ one small erosion $(<0.5 \mathrm{~mm})$, $2=$ two small or one large erosion $(>0.5 \mathrm{~mm}), 3=$ two or more large erosions and $4=$ any area of ulceration extending to the muscularis mucosa.

\section{Statistical analyses}

Data from all experiments are expressed as means with their standard errors. One-way ANOVA were used as appropriate. Where a significant effect was seen $(P<0 \cdot 05)$, individual comparisons were performed using $t$ tests based on the group means, residual and df obtained from ANOVA, a method equivalent to repeated-measures analyses. 

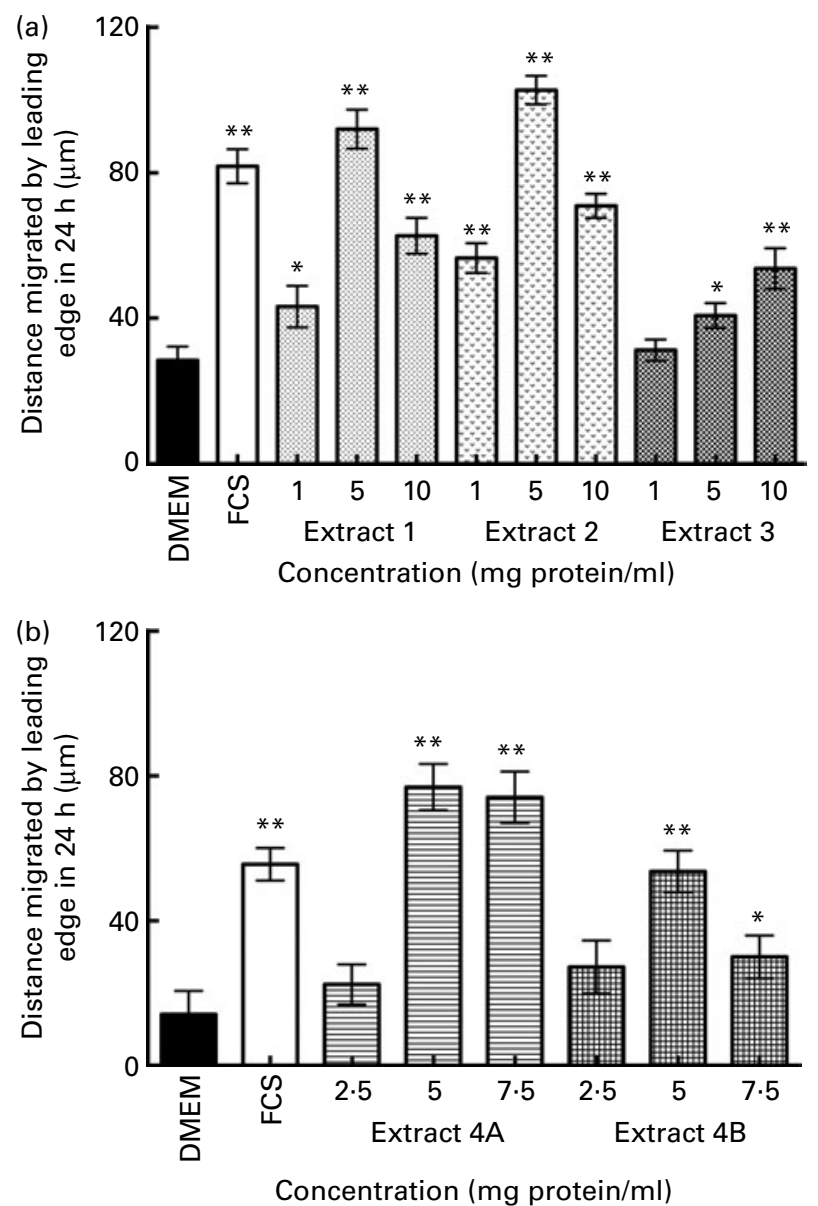

Fig. 1. Effect of Cordyceps sinensis (CS) on wound healing as assessed by cell migration. Serial photomicrographs of wounded monolayers of HT29 cells were taken. (a) Hot water extracts of whole (unground) rice- and potato-grown CS (extract 1), ground rice- and potato-grown CS (extract 2) and powdered liquid medium-grown CS (extract 3 ) were added to the cells. Negative control, Dulbecco's modified Eagle's medium (DMEM) alone; positive control, $10 \%$ fetal calf serum (FCS). (b) Extract 2 had a further preparation stage where it was mixed with ethanol $(1: 5, \mathrm{v} / \mathrm{v})$ at $4^{\circ} \mathrm{C}$ for $4 \mathrm{~h}$ The resulting precipitate was resuspended in distilled water (ethanol insoluble, extract 4A), and the liquid phase was decanted, dried, condensed and then resuspended in distilled water (ethanol soluble, extract 4B). Values are means, with standard errors represented by vertical bars. Mean values were significantly different from the negative control (DMEM): ${ }^{*} P<0.05$ and ${ }^{* *} P<0.01$

\section{Results}

Effect of the Cordyceps sinensis extracts on cell migration in vitro

Extract 1 (whole rice- and potato-grown hot water extract) and its ground equivalent (extract 2) gave pro-migratory effects with similar dose responses. Maximal effects were seen at a concentration of $5 \mathrm{mg} / \mathrm{ml}$ where a threefold increase in the rate of migration was demonstrated (Fig. 1(a)). The liquidphase-grown ground form also showed dose-dependent pro-migratory activity. The amount of pro-migratory activity was, however, significantly lower than the equivalent dose of ground rice- and potato-grown form $(P<0 \cdot 01) v$. equivalent dose for all three concentrations tested.
Analyses of ethanol solubility (samples 4A and B) showed that pro-migratory activity was present in both the ethanolsoluble and ethanol-insoluble fractions, with more activity being located in the ethanol-insoluble fraction (Fig. 1(b)).

As expected, these effects were not dependent on proliferation, as the presence of mitomycin $\mathrm{C}$ did not affect the rate of migration induced by CS (data not shown).

\section{Effect of the Cordyceps sinensis extracts on cell invasion}

Cells incubated in medium alone showed some degree of cell invasion through the collagen 1 gel after $48 \mathrm{~h}(5323 \pm 255$ relative fluorescence units). The presence of FCS, as a positive control, increased the number of cells progressing through the gel by $33 \%(7086 \pm 214$ relative fluorescence units, $P=0.003$; Fig. 2). At the highest concentration tested ( $5 \mathrm{mg}$ protein $/ \mathrm{ml}$ ), both the whole rice- and potato-grown hot water extract (extract 1) and its rice- and potato-grown ground equivalent (extract 2) also significantly increased the number of cells successfully invading through the gel $(P=0.013$ and $P=0.046$, respectively Fig. 2).

\section{Effect of the Cordyceps sinensis extracts on cell proliferation}

Whole-unground and potato-grown (extract 1), ground riceand potato-grown (extract 2) and liquid-phase-grown ground (extract 3) CS all showed pro-proliferative effects in a dose-dependent manner (Fig. 3(a)). For extracts 2 and 3, the optimal effect (threefold change over baseline) was seen at a concentration of $1 \mu \mathrm{g}$ protein $/ \mathrm{ml}(P=0.039$ for both; Fig. 3(a)). At concentrations above $0.01 \mathrm{mg}$ protein $/ \mathrm{ml}$, the presence of the extract began to reduce proliferation, but this may have been due to the onset of toxic effects. Similar results were seen with the whole-unground riceand potato-grown hot water extract (extract 1), although the

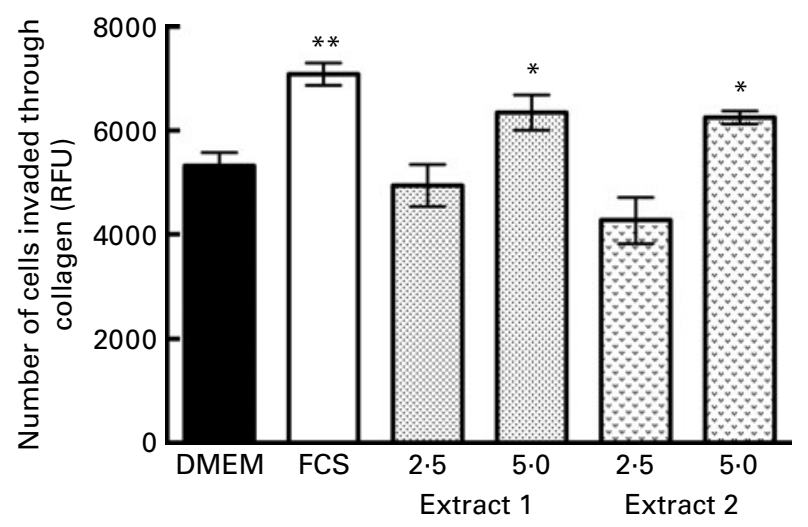

Concentration (mg protein/ml)

Fig. 2. Effect of the Cordyceps sinensis extracts on cell invasion. Extracts 1 and 2 were added to the cells and grown on the surface of collagen I gels for $48 \mathrm{~h}$. The number of cells reaching the inferior surface was determined fluorimetrically (relative fluorescence units, RFU). Symbols and positive and negative controls are similar to Fig. 1. DMEM, Dulbecco's modified Eagle's medium; FCS, fetal calf serum. Mean value was significantly different from that of the medium control: ${ }^{*} P<0.05,{ }^{* *} P<0.01$. 
(a)

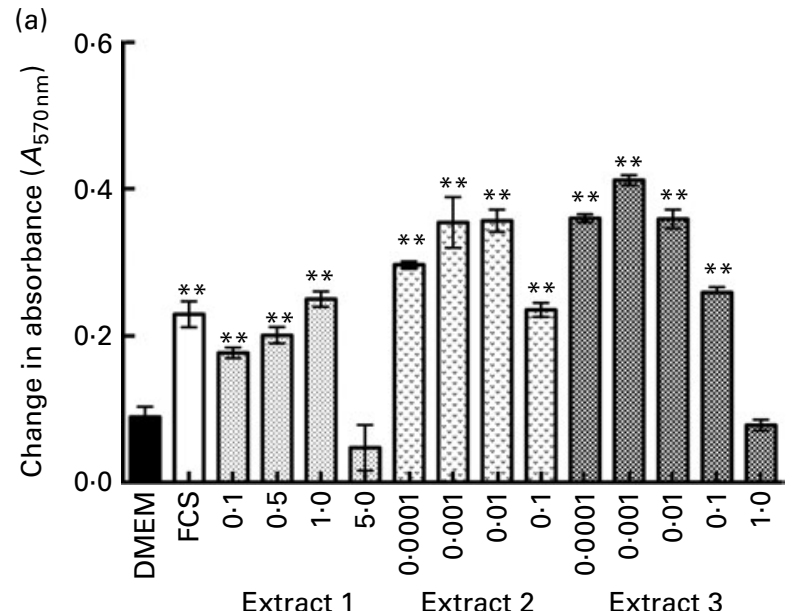

Concentration (mg protein $/ \mathrm{ml}$ )

(b)

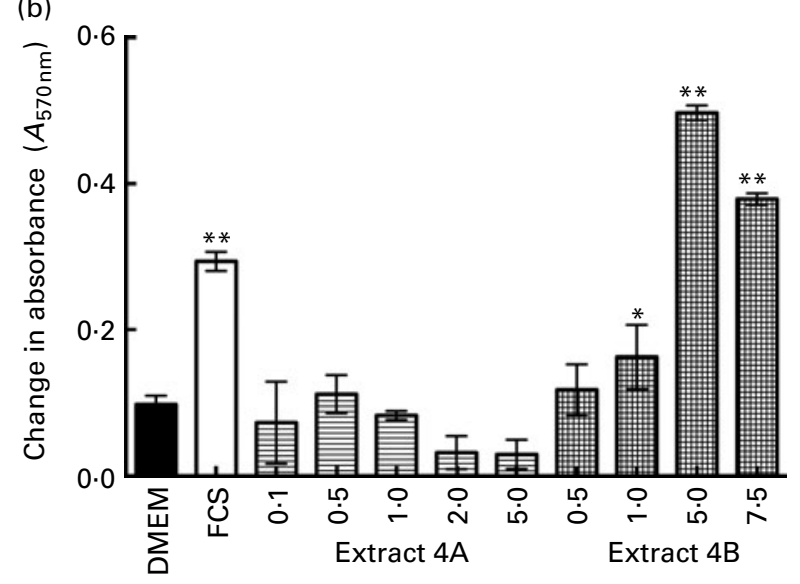

Concentration ( $\mathrm{mg}$ protein $/ \mathrm{ml}$ )

Fig. 3. Effect of Cordyceps sinensis on proliferation. (a) Extracts 1, 2, 3 and (b) extracts $4 \mathrm{~A}$ and 4B, as per Fig. 1, were added to HT29 cells. Cell proliferation assays utilised Alamar blue, measuring changes in absorbance at $570 \mathrm{~nm}$. Symbols and positive and negative controls are similar to Fig. 1 . DMEM, Dulbecco's modified Eagle's medium; FCS, fetal calf serum. Mean value was significantly different from that of the medium control: ${ }^{*} P<0.05$, ${ }^{\star *} P<0.01$.

concentrations required to stimulate proliferation were higher, with the maximal effect seen at $1 \mathrm{mg} / \mathrm{ml}$ (Fig. 3(a)).

Analyses of ethanol solubility (samples $4 \mathrm{~A}$ and $\mathrm{B}$ ) showed that pro-proliferative activity was only present in the ethanolsoluble fraction (Fig. 3(b)).

\section{Effect of the Cordyceps sinensis extracts on apoptosis}

Cells incubated with extract 1 (whole rice- and potato-grown hot water extract) or 2 (ground rice- and potato-grown hot water extract) in the absence of indomethacin showed values similar to those from cells incubated in medium alone (Fig. 4). Adding indomethacin to the cells caused a $35 \%$ rise $(P=0.037)$ in caspase- 3 activity. This result was not affected by the presence of both extracts. Changes in absorbance (used as a marker of caspase-3 activity) were shown to be specific, as these effects were not seen when the capase-3 inhibitor was also added to the cells (data not shown).

\section{Effect of the Cordyceps sinensis extracts on gastric damage in vivo}

Animals that received indomethacin and restraint and a placebo oral administration had a mean macroscopic gastric damage score of $47(\mathrm{SEM} 2.6) \mathrm{mm}^{2} /$ stomach. Administration of an epidermal growth factor, used as a positive control, reduced the amount of injury by $83 \%(P<0 \cdot 001$; Fig. 5). Oral administration with CS extract 2 or 3 showed similar dose-dependent reductions in macroscopic injury, with the higher dose $(20 \mathrm{mg}$ protein $/ \mathrm{ml})$ reducing damage by $58 \%$ (extract 2) and 60\% (extract 3) ( $P<0.001$; Fig. 5).

Histological assessment using the microscopic scoring system gave similar results to that obtained using macroscopic assessment of injury (data not shown). Gastric $\mathrm{pH}$ assessment showed that all animals had $\mathrm{pH}$ in the range of 1-3.

\section{Discussion}

On a regular basis, $72 \%$ of the American population use one or more health supplements, resulting in an annual turnover of $\$ 1.4$ billion ( $\$ 2.4$ billion) in the UK and $£ 10$ billion ( $\$ 18$ billion) in the USA, with $8 \%$ annual growth ${ }^{(17)}$. The sources of these 'natural' products are diverse and include bacteria, plant, animal, insect and marine origins ${ }^{(1)}$. Unfortunately, current evidence of the scientific validity of most of these compounds is severely limited. Nevertheless, some products have shown promising results for the treatment of gastrointestinal disorders, such as Iberogast (a mixture of nine herbs) ${ }^{(18)}$ and grape seed extract for the treatment of chemotherapyinduced mucositis ${ }^{(19)}$ and polyphenolic extracts from apple, which has been shown to prevent non-steroidal antiinflammatory drug-induced damage in the gut ${ }^{(20,21)}$.

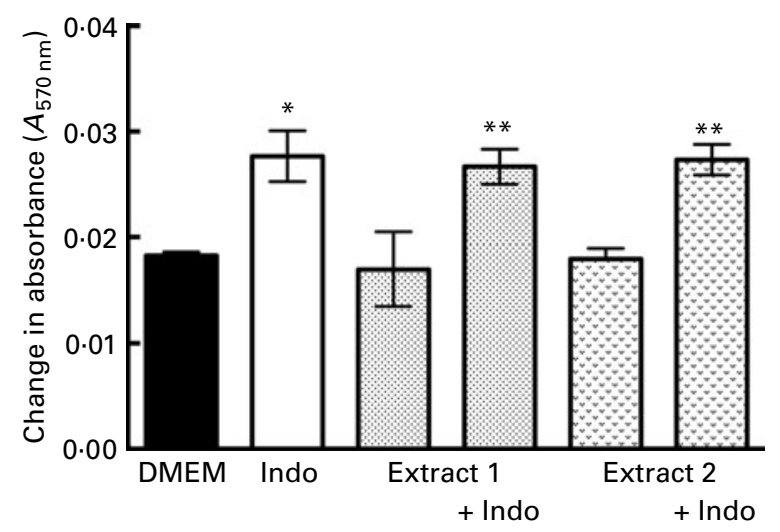

Fig. 4. (Lack of) Effect of the Cordyceps sinensis extracts on indomethacin (Indo)-induced apoptosis. HT29 cells were treated for $4 \mathrm{~h}$ with medium containing fetal calf serum alone or also containing Indo $(800 \mathrm{~mm}$, to stimulate apoptosis); additional wells included extract 1 or 2 (at $5 \mathrm{mg} / \mathrm{ml}$ ) with or with out Indo. Change in caspase- 3 activity was measured following absorbance at $405 \mathrm{~nm}$. Specificity of response was confirmed using the caspase-3 inhibitor Ac-DEVD-CHO (data not shown). Symbols are similar to Fig. 1. DMEM, Dulbecco's modified Eagle's medium. Mean value was significantly different from that of the medium control: ${ }^{\star} P<0.05,{ }^{\star *} P<0.01$. 


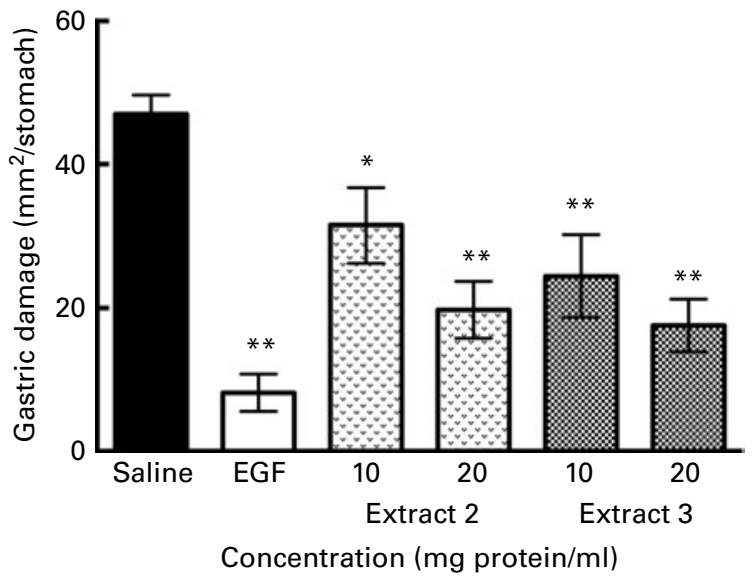

Fig. 5. Effect of Cordyceps sinensis on a rat model of gastric damage. Rats (eight animals per group) were given by oral administration $(2 \mathrm{ml})$ either saline (negative control), epidermal growth factor (EGF; $25 \mu \mathrm{g} / \mathrm{ml}$, positive control) or hot water Cordyceps sinensis extract 2 or 3 . After 30 min, animals received indomethacin $(20 \mathrm{mg} / \mathrm{kg}$, subcutaneously) and $3 \mathrm{~h}$ restraint. The extent of macroscopic damage was then determined. Values are means, with standard errors represented by vertical bars. Mean values were significantly different from those of the saline control, respectively: ${ }^{*} P<0.05$ and ${ }^{\star *} P<0.01$

We have shown that CS, which is commercially available through traditional Chinese medicine practitioners, in health food stores, and via the Internet, stimulates multiple aspects of gut mucosal integrity. It stimulated cell migration, invasion through collagen gel and proliferation in vitro and reduces the amount of gastric injury in rats.

For in vitro studies, human colonic (HT29) cells were used to examine the effects of CS on restitution, cell invasion, proliferation and apoptosis, as they provide robust reproducible models of human origin that we have experienced of using for the study of growth factors and other bioactive agents such as bovine colostrum ${ }^{(22,23)}$. Similarly, in vivo studies have examined the potential beneficial effect of CS on gastric (indomethacin- and restraint-induced) damage in rats, as non-steroidal anti-inflammatory drugs, such as indomethacin, continue to be a major cause of morbidity and mortality in humans ${ }^{(24)}$. Caution must be shown, however, in extrapolating results from in vitro cancer cell lines and animal models to the human situation.

Wound healing is generally characterised by processes involving migration and invasion, proliferation, and, in extensive injury, new blood vessel formation and remodelling. Our in vitro studies showed that the rice- and potato-grown and liquid-phase-grown CS possess pro-migratory, invasive and proliferative activity. The additional step of grinding the riceand potato-grown CS before undertaking hot water extraction had little, if any, value in enhancing restitutive or invasive activity but did increase its pro-proliferative effects. The liquid-phase-grown product was less potent in stimulating restitution compared with the ground rice- and potato-grown form but was comparable in terms of its pro-proliferative effects. Samples that had been processed to examine the alcohol solubility of active components showed that more than one compound was likely to be involved, as pro-migratory activity was present in both the ethanol-soluble and ethanolinsoluble fractions, whereas pro-proliferative activity was only present in the ethanol-soluble fraction.

Our in vivo study demonstrated protective effects of orally administered CS using a rat gastric damaging model. As gastric $\mathrm{pH}$ was not affected by CS administration, it can be described as a cytoprotective agent. Indomethacin causes damage to the gastrointestinal tract by several mechanisms including reduction of mucosal PG levels, reduction of mucosal blood flow, stimulating neutrophil activation and stimulating apoptosis $^{(25)}$. Additional work is required to determine which of these factors are influenced by CS, although our in vitro studies showing stimulation of cell migration and proliferation may have relevance. However, our in vitro studies suggested that the reduction in gastric injury seen was not due to the CS influencing apoptosis. While it remains possible that the gastroprotective effects seen were mediated through topical physico-chemical actions, the results from our in vitro studies suggested that protective mechanisms, such as stimulation of restitution, may have been mediated through pharmacological actions.

Previous biochemical analyses on CS have suggested that the major potential bioactive components within CS are nucleoside adenosine, nucleotide adenosine monophosphate and an ethanol-insoluble polysaccharide comprising a $13-\beta$-D-glucan with some $1 / 6$ branch chain ${ }^{(26)}$. This polysaccharide component of CS has previously been reported to have immunomodulatory effects, stimulating mouse monocyte activation in vitro ${ }^{(26)}$. Hot water extract of CS has also been reported to partially protect mice against the damaging effect of high-dose radiation on bone marrow and intestinal injury $^{(27)}$. The mechanism through which this worked was unclear, although in vitro studies using the osteoblastic cell line MC3T3-E1 have suggested that CS extract may act as a free radical scavenger ${ }^{(27)}$.

Our finding of the disassociation between proliferative and migratory activity suggests that more than one compound is involved in the current series of studies. The nucleotide and nucleoside content of CS (which would remain in the ethanol-soluble sub-fraction) may be relevant, as we demonstrated previously pro-proliferative, pro-migratory and gastric injury protective effects using pure adenosine compounds ${ }^{(28)}$. Furthermore, based on published quantitative analyses studies $^{(29)}$, the amount of nucleotides/nucleosides present in the extracts used is sufficient to account for the pro-proliferative effects and in the same order of magnitude as that demonstrated by us previously to elicit pro-migratory effects ${ }^{(28)}$. Although the vast majority of pro-proliferative activity was seen in the ethanol-soluble sub-fraction, migratory activity was also seen in the ethanol-insoluble component. The polysaccharide component of CS mentioned earlier would be within this subfraction and may, therefore, have relevance to this finding.

3-Deoxyadenosine, known as cordycepin, was originally isolated from Cordyceps species and has been investigated by others ${ }^{(30)}$. This purine nucleoside antimetabolite is now produced synthetically and possesses potential anti-neoplastic activity through inhibiting transcription elongation and RNA synthesis due to the absence of a hydroxyl moiety at the 
$3^{\prime}$ position. The concentration of cordycepin within CS is, however, much lower than materials mentioned above ${ }^{(31)}$ and unlikely to be relevant to the effects seen.

Many of the general public consider that natural-based products equate to a gentle response, as distinct from a pharmaceutical approach ${ }^{(17)}$. It is, therefore, of note that the doses of CS used in the rat gastric damage study (10 and $20 \mathrm{mg} / \mathrm{ml}$ ) gave similar results to those seen in animals given the potent cytoprotective agent epidermal growth factor administered at $25 \mu \mathrm{g} / \mathrm{kg}$. Furthermore, the concentrations of CS used in our restitution studies $(5 \mathrm{mg} / \mathrm{ml})$, and in our gastric damage model $(10-20 \mathrm{mg} / \mathrm{ml})$, are likely to be present in the human gastric juice of subjects taking CS supplements, as the standard recommended dose is between 650 and $3000 \mathrm{mg}$, taken twice a day, with the average person producing a resting gastric juice volume of $150 \mathrm{ml} / \mathrm{h}^{(32)}$. These results further emphasise that the division between 'food products' and 'drugs', when considered in terms of biological activity, is far from clear and that these products should be considered as functional foods. The regulatory control of such food products is, however, much less stringent than if labelled as a pharmaceutical product.

In conclusion, our studies have shown that CS, commercially available as over-the-counter 'health foods' for licensing purposes, possesses biological activity at clinically relevant doses when assessed using several models of gut integrity and repair.

\section{Acknowledgements}

CS was provided by importers Neovite UK Limited, Dulwich Wood Park, London, UK. R. J. P., E. O., C. J. P. and T. M. have no conflict of interest. The present study received no specific grant from any funding agency in the public, commercial or not-for-profit sectors. R. J. P. and T. M. contributed to the design of the experiment, analysis of the results and writing of the manuscript; E. O. performed the experiments and analysis of the results; C. J. P. performed the experiments and analysis of the results.

\section{References}

1. Ghosh S \& Playford RJ (2003) Bioactive natural compounds for the treatment of gastrointestinal disorders. Clin Sci 104, $547-556$

2. Dong CH \& Yao YJ (2005) Nutritional requirements of mycelial growth of Cordyceps sinensis in submerged culture. J Appl Microbiol 99, 483-492.

3. Zhong JJ \& Xiao JH (2009) Secondary metabolites from higher fungi: discovery, bioactivity, and bioproduction. Adv Biochem Eng Biotechnol 113, 79-150.

4. Chen Y, Guo H, Du Z, et al. (2009) Ecology-based screen identifies new metabolites from a Cordyceps-colonizing fungus as cancer cell proliferation inhibitors and apoptosis inducers. Cell Prolif 42, 838-847.

5. Chen S, Li Z, Krochmal R, et al. (2010) Effect of Cs-4 (Cordyceps sinensis) on exercise performance in healthy older subjects: a double-blind, placebo-controlled trial. J Altern Complement Med 16, 585-590.
6. Koh JH, Kim KM, Kim JM, et al. (2003) Antifatigue and antistress effect of the hot-water fraction from mycelia of Cordyceps sinensis. Biol Pharm Bull 26, 691-694.

7. Yamaguchi Y, Kagota S, Nakamura K, et al. (2000) Inhibitory effects of water extracts from fruiting bodies of cultured Cordyceps sinensis on raised serum lipid peroxide levels and aortic cholesterol deposition in atherosclerotic mice. Phytother Res 14, 650-652.

8. Colson SN, Wyatt FB, Johnston DL, et al. (2005) Cordyceps sinensis- and Rhodiola rosea-based supplementation in male cyclists and its effect on muscle tissue oxygen saturation. J Strength Cond Res 19, 358-363.

9. Fogh J, Fogh JM \& Orfeo T (1977) One hundred and twentyseven cultured human tumor cell lines producing tumors in nude mice. J Natl Cancer Inst 59, 221-226.

10. Brown RE, Jarvis KL \& Hyland KJ (1989) Protein measurement using bicinchoninic acid: elimination of interfering substances. Anal Biochem 180, 136-139.

11. Marchbank T, Mahmood A, Fitzgerald AJ, et al. (2007) Human pancreatic secretory trypsin inhibitor stabilizes intestinal mucosa against noxious agents. Am J Pathol 171, $1462-1473$.

12. Marchbank T, Weaver G, Nilsen-Hamilton M, et al. (2009) Pancreatic secretory trypsin inhibitor is a major motogenic and protective factor in human breast milk. Am J Physiol Gastrointest Liver Physiol 296, G697-G703.

13. Nakayama GR, Caton MC, Nova MP, et al. (1997) Assessment of the Alamar Blue assay for cellular growth and viability in vitro. J Immunol 204, 205-208.

14. Fitzgerald AJ, Rai PS, Marchbank T, et al. (2005) Reparative properties of a commercial fish protein hydrolysate preparation. Gut 54, 775-781.

15. Playford RJ, Belo A, Poulsom R, et al. (2006) Effects of mouse and human lipocalin homologues $24 \mathrm{p} 3 / \mathrm{lcn} 2$ and neutrophil gelatinase-associated lipocalin on gastrointestinal mucosal integrity and repair. Gastroenterology 131, 809-817.

16. Playford RJ, Vesey DA, Haldane, et al. (1991) Dosedependent effects of fentanyl on indomethacin-induced gastric damage. Digestion 49, 198-203.

17. Sloan AE (2002) The top 10 functional food trends: the next generation. Food Technol 56, 32-57.

18. Wright TH, Yazbeck R, Lymn KA, et al. (2009) The herbal extract, Iberogast, improves jejunal integrity in rats with 5-fluorouracil (5-FU)-induced mucositis. Cancer Biol Ther 8, 923-929.

19. Cheah KY, Howarth GS, Yazbeck R, et al. (2009) Grape seed extract protects IEC-6 cells from chemotherapy-induced cytotoxicity and improves parameters of small intestinal mucositis in rats with experimentally-induced mucositis. Cancer Biol Ther 8, 382-390.

20. Graziani G, D’Argenio G, Tuccillo C, et al. (2005) Apple polyphenol extracts prevent damage to human gastric epithelial cells in vitro and to rat gastric mucosa in vivo. Gut 54, 193-200

21. D'Argenio G, Mazzone G, Tuccillo C, et al. (2008) Apple polyphenol extracts prevent aspirin-induced damage to the rat gastric mucosa. Br J Nutr 100, 1228-1236.

22. Playford RJ, Floyd DN, Macdonald CE, et al. (1999) Bovine colostrum is a health food supplement which prevents NSAID induced gut damage. Gut 44, 653-658.

23. Mahmood A, FitzGerald AJ, Marchbank T, et al. (2007) Zinc carnosine, a health food supplement that stabilises small bowel integrity and stimulates gut repair processes. Gut 56, 168-175. 
24. Bjarnason I, Zanelli G, Prouse P, et al. (1987) Blood and protein loss via small intestinal inflammation induced by nonsteroidal antiinflammatory drugs. Lancet ii 711-714.

25. Levi S \& Shaw-Smith C (1994) Non-steroidal anti-inflammatory drugs: how do they damage the gut? Br J Rheumatol 33, 605-612.

26. Akaki J, Matsui Y, Kojima H, et al. (2009) Structural analysis of monocyte activation constituents in cultured mycelia of Cordyceps sinensis. Fitoterapia 80, 182-187.

27. Liu WC, Wang SC, Tsai ML, et al. (2006) Protection against radiation-induced bone marrow and intestinal injuries by Cordyceps sinensis, a Chinese herbal medicine. Radiat Res 166, 900-907.

28. Belo A, Marchbank T, Fitzgerald A, et al. (2006) Gastroprotective effects of oral nucleotide administration. Gut 55, 165-171.
29. Leung PH, Zhang QX \& Wu JY (2006) Mycelium cultivation, chemical composition and antitumour activity of a Tolypocladium sp. fungus isolated from wild Cordyceps sinensis. J Appl Microbiol 101, 275-283.

30. Cunningham KG, Manson W, Spring FS, et al. (1950) Cordycepin, a metabolic product isolated from cultures of Cordyceps militaris (Linn.). Nature 166, 949.

31. Guo FQ, Li A, Huang LF, et al. (2006) Identification and determination of nucleosides in Cordyceps sinensis and its substitutes by high performance liquid chromatography with mass spectrometric detection. J Pharm Biomed Anal 40, 623-630.

32. Playford RJ, Batten JJ, Freeman TC, et al. (1991) Gastric output of pancreatic secretory trypsin inhibitor is increased by misoprostol. Gut 32, 1396-1400. 